\title{
Cystic Fibrosis Serum Effect on the Short Circuit Current of Rat Jejunum
}

\author{
ROBERT D. TUCKER, G. E. GIBBS, AND M. B. CHRISTENSEN \\ Department of Pediatrics, University of Nebraska College of Medicine, Omaha, Nebraska, USA
}

\begin{abstract}
Summary
Sera from 100 subjects were tested for the presence of the cystic fibrosis (CF) "factor" utilizing the short circuit current rat jejunum bioassay. Comparing the mean percent decrease in short circuit current, the presumed normal group differs from the homozygous CF group at $P=0.001$ and from the heterozygous group at $P=$ 0.05. The two CF genotypes are statistically different at $P=0.004$. There is, however, a large overlap among the three groups, which limits the assay's utility as a guide for factor purification as well as clinical use.
\end{abstract}

\section{Speculation}

Serum and saliva from CF genotypes have been shown to contain a factor (or factors) which decreases the sodium ion transport across the cell membrane. Currently, a bioassay is being sought to guide isolation of the factor for development of a radioimmunoassay. Characterization of the factor may explain the underlying transport error in $\mathrm{CF}$ as well as enable heterozygote carrier identification.

Numerous assays have been proposed for detection of the CF factor, including ciliary dyskinesia in various ciliated tissues, inhibition of sodium reabsorption in the rat parotid gland and changes in electrical characteristics of rat jejunum $(1,3,5,9,11)$. The ciliary assays have been used in attempts to purify and characterize the factor, which is needed for development of a radioimmunoassay. Progress toward achievement of purification and characterization has been limited by inadequacy of the available assay methods. Meanwhile, there is possibility that a bioassay could find some use directly in genetic counseling.

As early as 1967, Spock et al. (11) reported the existence of a factor present in the serum of both heterozygotes and homozygotes, but not in normal genotypes, which destroyed the usual beat synchronization of rabbit tracheal cilia in vitro. The presence of a serum ciliary dyskinesia factor was also reported by several other groups utilizing an oyster and a mussel gill cilia assay $(3,5)$. These results, however, have not been consistent within each laboratory, or reproducible from laboratory to laboratory, presumably due to seasonal variation and infection $(6,10)$. Utilizing guanidine gel filtration and guided by the oyster gill assay, a partial purification has led to characterization of the factor as a small polypeptide of molecular weight between 6000 and 11,000 (4).

In order to establish a more reliable bioassay, Araki et al.(1) adapted the short circuit current (SCC) procedure. The assay procedure, modified by Araki et al. (2) in 1976, consists of establishing the SCC of an isolated section of rat jejunum while both the mucosal and serosal sides are bathed with oxygenated Krebs' Ringer's solution. The mucosal solution is then replaced with a serum-Krebs' Ringer's solution and the SCC is again determined. Araki et al. hypothesize that the observed decrease in SCC caused by $\mathrm{CF}$ genotype serum is due to interference by the abnormal CF factor with glucose metabolism, which, in turn, reduces active sodium transport.

The present communication consists of a further exploration of the effect of CF and normal genotype sera on the short circuit current of rat jejunum.

\section{MATERIALS AND METHODS}

\section{EQUIPMENT}

A specially designed lucite chamber, similar to a Ussing chamber (12), was constructed to hold the rat jejunum. The tissue is mounted as a flat sheet between the two matching lucite half chambers and held firmly in place by six pins. The membrane is protected from extensive pressure damage by 0.02 in thick silastic sheets mounted on the two half-cell chamber mating surfaces. Each side of the chamber holds approximately $1 \mathrm{cc}$ of solution and is connected to a separate perfusion column. The individual perfusion columns circulate, at a rate of $1-2 \mathrm{cc} / \mathrm{sec}$, a total volume of about $12 \mathrm{cc}$, by a $95 \% \mathrm{O}_{2}-5 \% \mathrm{CO}_{2}$ gas lift. The temperature of the circulating solutions is maintained at $37^{\circ} \mathrm{C}\left( \pm 0.2^{\circ} \mathrm{C}\right)$ by a water jacket encircling the columns.

The transmembrane potential difference is measured differentially by connecting two standard calomel glass electrodes to the separate sides of the lucite chamber via Ringer's-3\% agar salt bridges. These salt bridges decrease chamber size, minimize the junction potential at the tip of the calomel half-cells, and decrease any possibility of electrode fibertip contamination. The electrodes input to a Burr Brown instrumentation differential amplifier. This FET-input amplifier was chosen for its high input impedance, temperature stability, and high common mode rejection. The amplifier output is continuously displayed on one channel of a Honeywell two-channel strip recorder as well as on a digital multimeter.

Any constant current, from 1 microampere to over 10 milliamperes, can be driven through the membrane by way of two pure platinum electrodes mounted in the end of each lucite half-cell. The current driver readings are displayed on a second digital multimeter and the second channel of the strip chest recorder. The second channel also doubles as a monitor of the temperature of the membrane-bathing solutions. This channel records the solution temperature via a YSI probe placed in one of the perfusion columns, except during current measurements, at which time the channel is switched to plot the current driver output.

\section{PROCEDURE}

Male Sprague-Dawley rats weighing between $250-300 \mathrm{~g}$ are anesthetized by ip injection of sodium pentobarbital. The small bowel is exposed through a midline incision and the arcade arteries of a small section of jejunum, approximately $2 \mathrm{~cm}$ in length, are clamped. This section is then removed and placed in a Petri dish of mammalian Ringer's solution, whereupon it is opened along the mesenteric border and washed free of intestinal contents.

After tissue mounting, the transmembrane potential is allowed 
to stabilize in mammalian Krebs' Ringer's solution containing 15 $\mathrm{mM} /$ liter dextrose. Stabilization usually occurs within 5-15 min. Every $2 \mathrm{~min}$, for a total of 10 measurements, the transmembrane potential is driven to zero by the current driver, thereby allowing calculation, via Ohm's law, of the resistance between the voltagesensing electrodes. Serum is then added to the perfusion column on the mucosal side of the jejunum, so that the bathing solution is comprised of Kreb's Ringer's and serum in a 3:1 ratio. For selected sera, stronger dilution ratios of $2: 1$ and $1: 1$ were also compared with results obtained with 3:1 dilutions; these initial experiments yielded no significant differences between the two dilutions. Characteristically, the transmembrane potential decreases after serum addition but becomes stable again at a lower value within several min. Again, 10 measurements are performed, as outlined above, to permit calculation of the total resistance between the calomel electrodes.

The system resistance, that is, the resistance of the solution between the calomel electrodes, is found by recording the current needed to cause a known potential change without the tissue in place. The membrane resistance, before ( 10 measurements) and after ( 10 measurements) the addition of sera, can be calculated by subtracting the system resistance from the total resistance. The short circuit currents are then calculated by dividing the voltage obtained immediately before each of the 20 measurements by the corresponding membrane resistance values.

\section{RESULTS}

Representative plots obtained as an output from the Honeywell strip chart recorder are shown in Figures 1 and 2: Figure 1 shows the data from a CF homozygous serum and Figure 2 the results from a normal serum. In both figures, the top tracing shows the transmembrane potential as a function of time, both before and after addition of a unknown serum. Full scale represents $10 \mathrm{mv}$, mucosal solution negative with respect to serosal.

The middle and bottom traces were both plotted by the second channel of the Honeywell recorder. The temperature of the bathing solution was monitored until current measurements were performed and is represented by the middle tracing. At this point, channel no. 2 was switched from the temperature transducer to the output of the current driver. The current necessary to drive the transmembrane potential to zero is shown as a vertical line for each of the 20 readings.

At the end of the experimental run, the tissue was removed and the DC offset voltage was obtained. This small bias, always less than $0.2 \mathrm{mv}$, was, of course, subtracted from the appropriate voltage measurements.

The system resistance was found by passing a measured current to achieve a transmembrane potential difference of $8 \mathrm{mv}$. The system resistance-determining voltage and current measurements were plotted as vertical lines at the end of the experimental run and are indicated on both figures (e.g., $8 \mathrm{mv}$ and 309 microA in Fig. 1).

The SCC as a function of time is calculated from these strip chart recorder outputs. Figure 3 shows the time course of the SCC for a presumed normal serum and a homozygous CF serum, in which the normal serum led to a small decrease in the SCC while the CF serum caused a dramatic drop.

Because the SCC values vary among tissue samples, the percent decrease in the averaged SCC (the ratio of the average of the 10

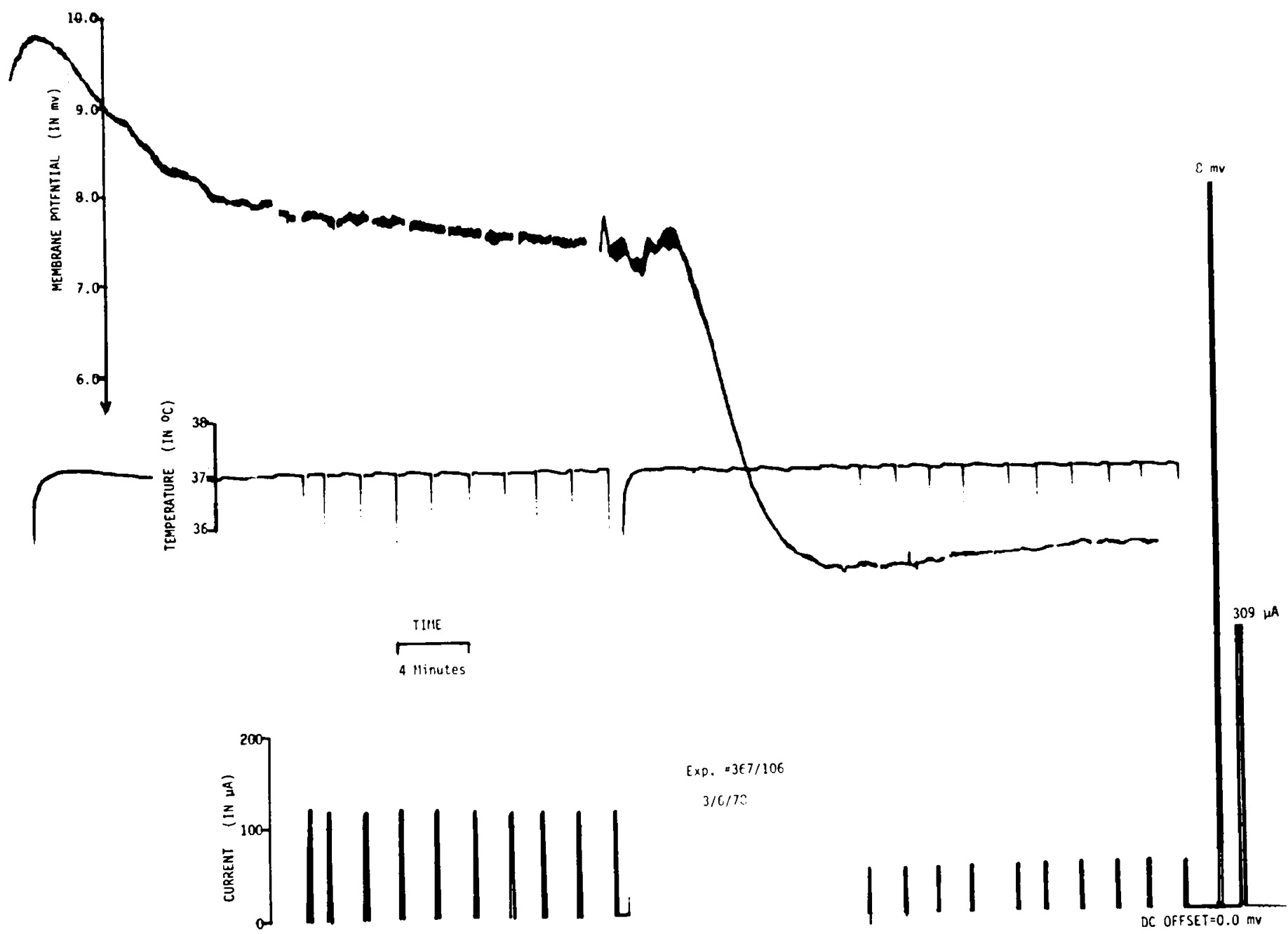

Fig. I. Original strip chart recorder output for an experiment with CF homozygous serum, which shows a large decrease in SCC. 


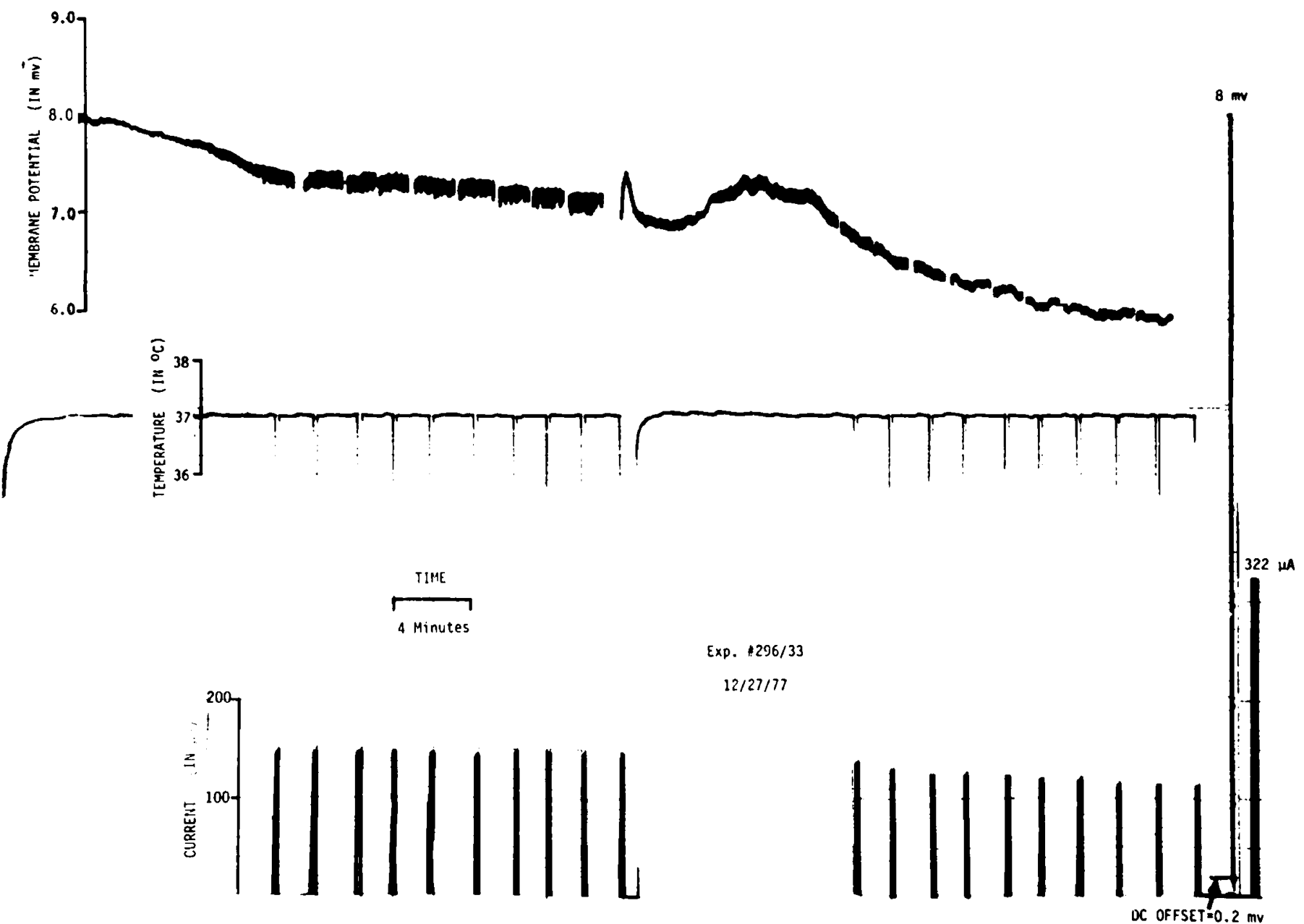

Fig. 2. Original strip chart recorder output for an experiment with presumed normal serum, which shows little change in SCC.

SCC readings obtained after the serum addition to the average of the 10 values measured before the addition of the serum) is employed as a discriminator among normal and CF genotypes. Qualitatively, all the data exhibit similar curve shapes such as those shown in Figure 3, but, unfortunately, there is a range of normal values which encompasses the entire range of the CF genotypes.

Figure 4 summarizes the results of 100 blind experiments on 42 presumed normals, 32 obligate CF heterozygotes, and $26 \mathrm{CF}$ homozygotes. In the disease state, the data tend to be clustered at the higher values; however, the control group also displays a significant number of sera testing with a large percent decrease in SCC. Whereas it is true that 1 in 20 presumed normals are probably heterozygous, this cannot statistically account for the large number of normals testing as CF genotypes. In the sample size in this study, one would expect only two or three presumed normals, in actuality CF heterozygotes, to appear as false positives whereas, in fact, 17 normal sera exhibited a greater than $40 \%$ decrease in SCC.

The means of the three groups are compared employing standard statistical analysis. The means and SEM's are indicated on Figure 4. The average percent decrease in SCC of the normal group, mean of 30.9, differs from the heterozygous group mean of 38.9 at $P=0.05$ and from the homozygous group mean of 50.6 at $P=0.001$. The CF genotypes, heterozygous $v$ s. homozygous, are statistically different at $P=0.004$. (These $P$ values represent twotailed hypothesis tests).

In order to ascertain some measure of the variation in the assay, a series of blind experiments were conducted on 10 presumed normals and four CF homozygous subjects. Multiple determinations, from two to nine, were performed on each subject's serum.

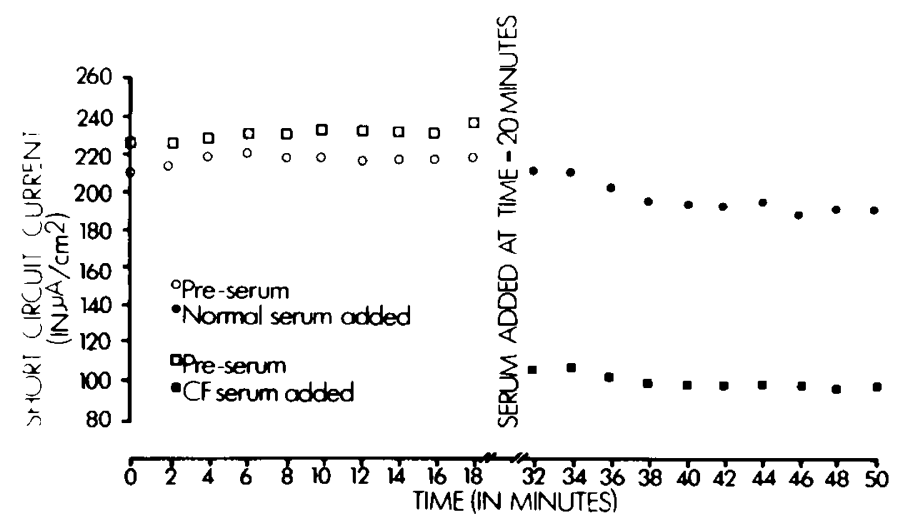

Fig. 3. Short circuit current $v s$. time for two representative experiments, a presumed normal serum and a CF homozygous serum.

(At least three runs were attempted on all 16 subjects.) All homozygous CF genotypes tested positive in every experiment, whereas four of the presumed normals also exhibited one positive experiment. Of these four presumed normals, three experiments were conducted on each of three subjects and nine experiments on the other subject. Such variation would, of course, limit the clinical utility of the bioassay as a one determination test.

\section{DISCUSSION}

The results of the short circuit current method, adapted by Araki et al. $(1,2)$ as a cystic fibrosis factor assay, were confirmed by Gilmore $e t$ al. (8) on a small number of normals, heterozygotes, 


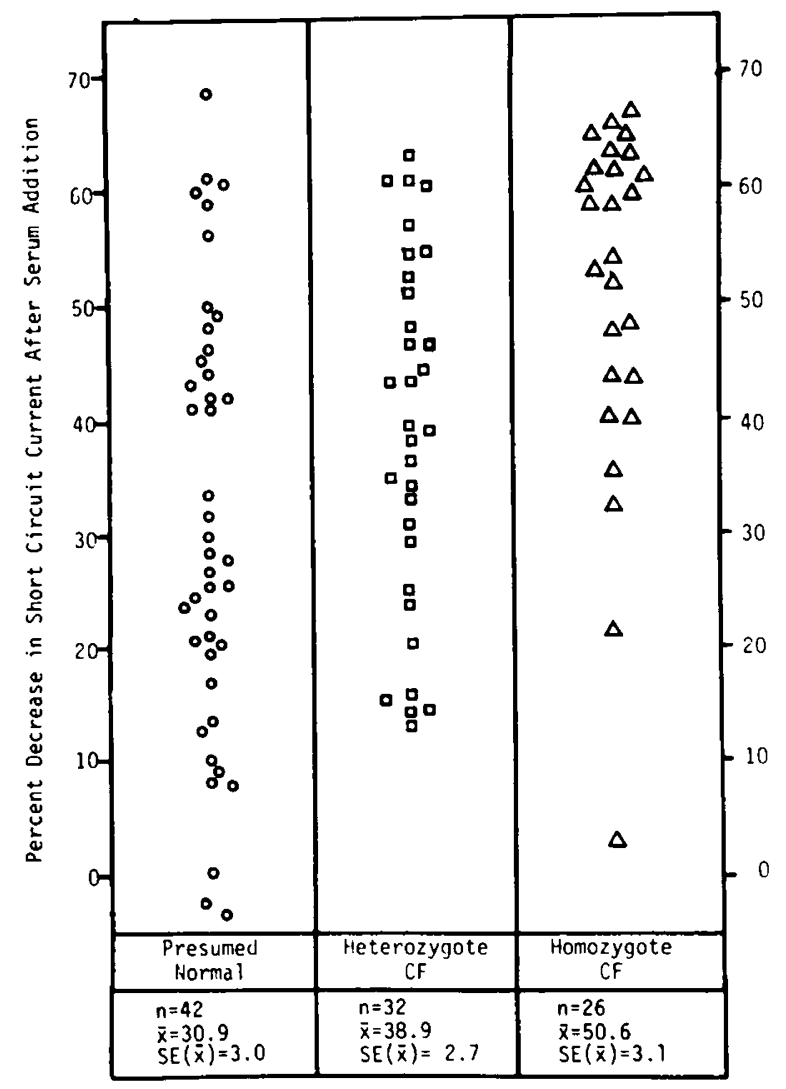

Fig. 4. The percent decrease in SCC after serum addition for the three experimental groups. The number of sera tested $(n)$, the mean $(\bar{x})$ and the standard error of the mean $(S E(\bar{x}))$ are indicated below the corresponding groups.

and homozygotes. These studies suggest that the CF factor is heat labile, is present in the serum of both CF genotypes, and that it dramatically reduces the SCC.

The data which have been presented here do not, however, totally confirm these earlier results. Whereas the homozygous $C F$ group is statistically significantly different, at a high degree of reliability, from the group of presumed normals, the data display considerably more false positives than can be expected by pure chance. It is possible that the number of presumed normals which exhibit large decreases in SCC, are influenced by factors other than CF genotype. Conover et al. (7) have found evidence for the cilia toxic factor in patients with autoimmune and allergic diseases. Three individuals in the control group of the present study showing large decreases in SCC were later found to have a history of allergy and there may have been others which were not recognized.
The data of this study differs from those of Araki et al. $(1,2)$ and of Gilmore et al. (8) in the effect of heterozygous serum on SCC. The group mean and data distribution of the obligate heterozygotes is more similar to the presumed normal group than to the homozygous CF group. The small number of subjects tested in the previous studies may account, in part, for the difference between results of the present study from the previous ones.

Whereas the SCC assay may prove useful in the further study of $\mathrm{CF}$ and may, indeed, yield more information concerning the relation between $C F$ and immunologic diseases, the assay system does not at this time appear to be clinically useful in distinguishing heterozygote CF genotypes. Therefore, the authors conclude that currently the SCC assay must be considered, at best, a laboratory research tool rather than a clinical test.

\section{REFERENCES AND NOTES}

1. Araki, H., Field, M., and Shwachman. H.: A new assay for cystic fibrosis factor: Effects of sera from patients with cystic fibrosis on the in vitro electrical properties of rat jejunum. Pediatr. Res., 9: 932 (1975).

2. Araki, H., Field, M., and Shwachman, H.: A new assay for cystic fibrosis factor: Effect of sera from patients with cystic fibrosis on the in vitro electrical properties of rat jejunum. Abstracts of Medical Sessions VII International Cystic Fibrosis Congress, Paris, France, p. 28 (1976).

3. Besley, G. T. N., Patrick, A. D., and Norman, A. P.: Inhibition of the mortality of gill cilia of dreissensia by plasma of cystic fibrosis patients and their parents. J. Med. Genet., 6: 278 (1969).

4. Bowman, B. H., Lankford, B. J., Fuller, G. M., Carson, S. D., Kurosky, A., and Barnett, D. R.: Cystic fibrosis: the ciliary inhibitor is a small polypeptide associated with immunoglobulin G. Biochem. Biophys. Res. Commun., 64: 1310 (1975).

5. Bowman, B. H., Lockhart, L. H., and McCombs, M. L.: Oyster ciliary inhibition by cystic fibrosis factor. Science, 164: 325 (1969).

6. Cherry, J. D., Roden, V. S., Regent, A. J., and Dorner, R. W.: The inhibition of ciliary activity in tracheal organ cultures by sera from children with cystic fibrosis and control subjects. J. Pediatr., 79: 937 (1971).

7. Conover, J. H., Conod, E. J., and Hirschhorn, K.: Ciliary dyskinesia factor in immunological and pulmonary disease. Lancet, 1 : 1194 (1973).

8. Gilmore, J. P., Davis, M., and Gibbs, G. E.: Influence of cystic fibrotic and heterozygous serum on rat jejunum. Proc. Soc. Exp. Biol. Med., 157: 70 (1978).

9. Mangos, J. A., McSherry. N. R., and Benke, P. J.: A sodium transport inhibitory factor in the saliva of patients with cystic fibrosis of the pancreas. Pediatr. Res., 1: 436 (1967).

10. Posselt, H., Bender, S.: Heterozygote testing in cystic fibrosis. Z. Kinderheilkund. 100: 93 (1971).

11. Spock, A., Heick, H. M. C., Cress, H., and Logan, W. S.: Abnormal serum factor in patients with cystic fibrosis of the pancreas. Pediatr. Res., 1: 173 (1967).

12. Ussing, H. H., and Windhager, E. E.: Nature of shunt path and active sodium transport through frog skin epithelium. Acta Physiol. Scand., 61: 484 (1964).

13. Informed consent was obtained from patients or parents for these studies.

14. The present address of Dr. Robert D. Tucker is: Box 511 Mayo Building. University of Minnesota, Minneapolis, MI 55455 (USA).

15. The authors thank Deborah Lindquist and Mrs. Doris Raasch.

16. This research was supported by a special appropriation from the Nebraska State Legislature, also by grants from the American Lung Association of Nebraska and the Cystic Fibrosis Foundation.

17. Requests for reprints should be addressed to: Dr. Gordon E. Gibbs. Department of Pediatrics, University of Nebraska College of Medicine, 42nd and Dewey Avenue. Omaha, NE 68105 (USA).

18. Received for publication September 19, 1978

19. Accepted for publication January 11, 1979. 